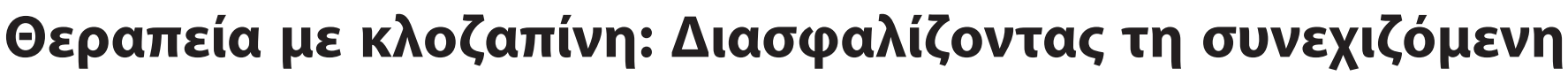

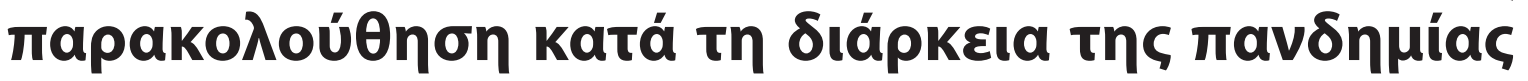

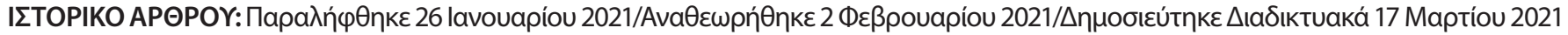

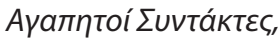

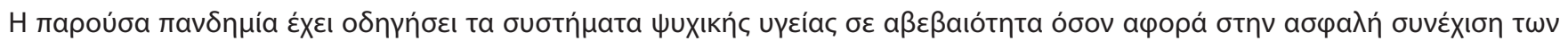

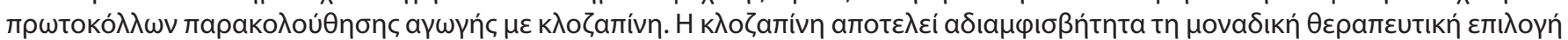

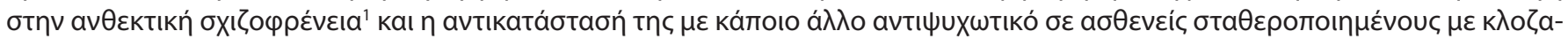

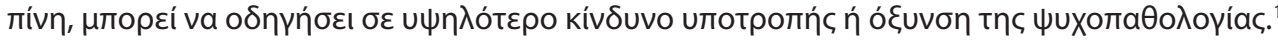

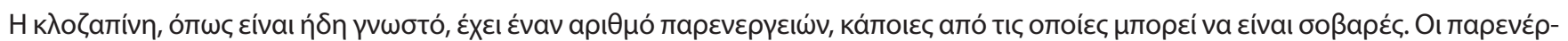

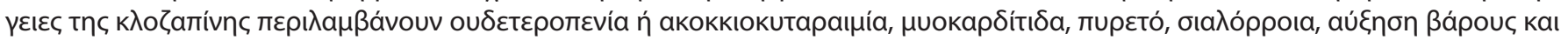

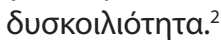

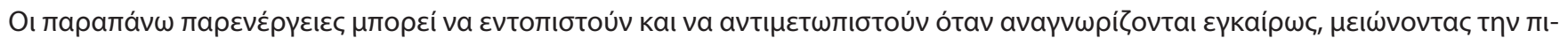

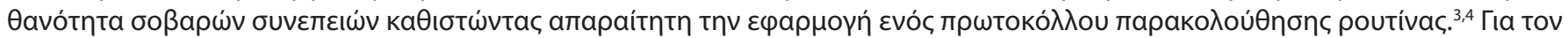

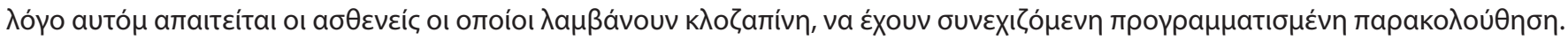

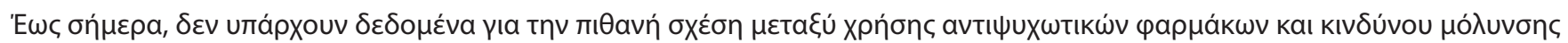

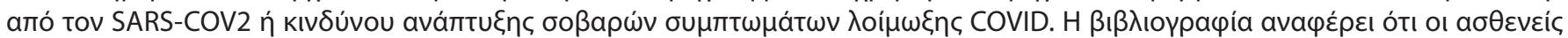

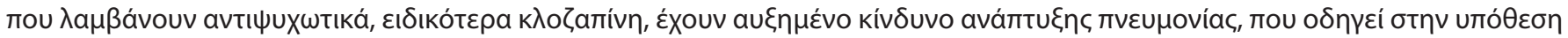

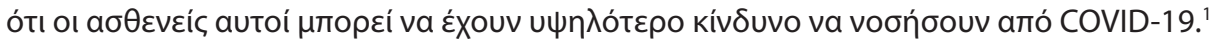

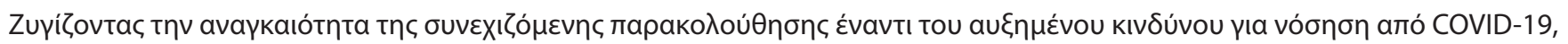

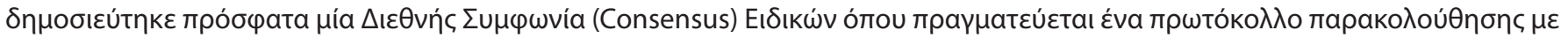

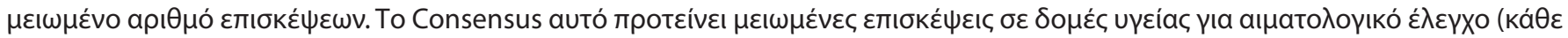

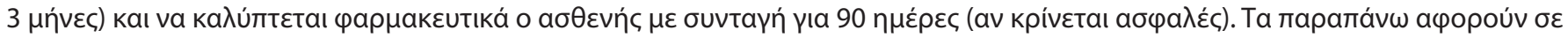

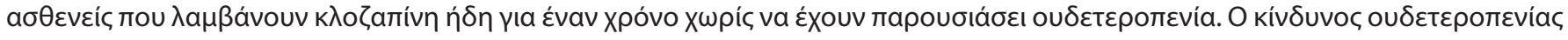

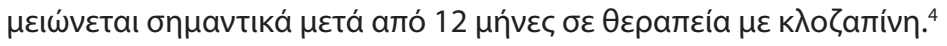

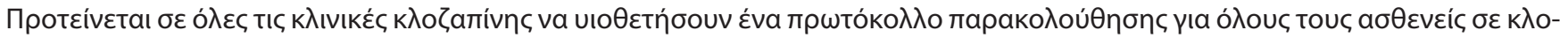

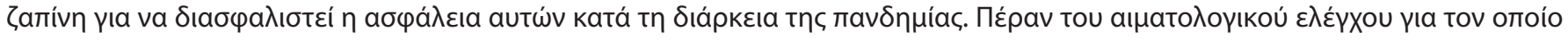

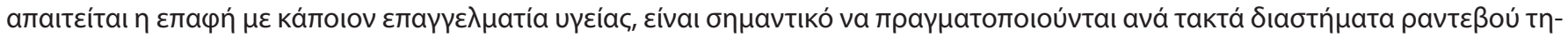

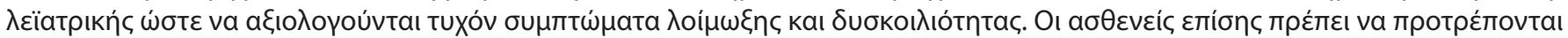

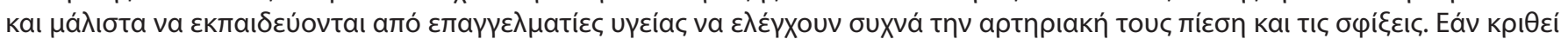

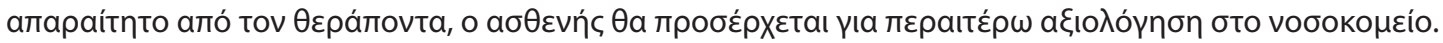

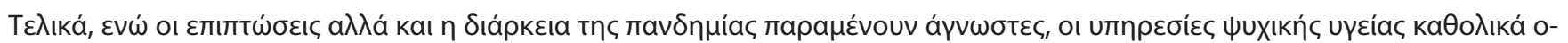

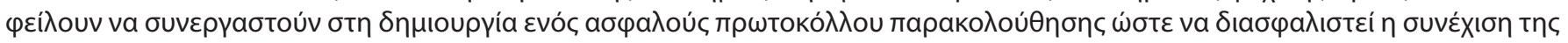

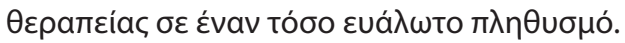

\section{Mapía Nuota〈ákn}

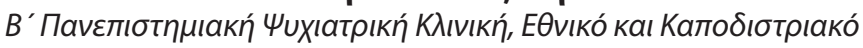

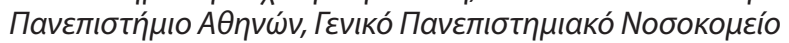
AтtıKóv, AӨńva, e-mail: manystazaki@nurs.uoa.gr

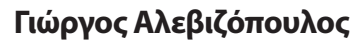

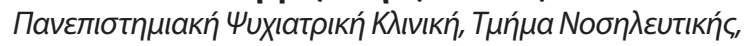

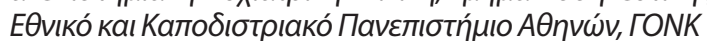
Oı'Ay.Avápyupol, AӨńva, e-mail:galev@nurs.uoa.gr

\section{References}

1. Siskind D, McCartney L, Goldschlager R, Kisely S. Clozapine v. first- and second-generation antipsychotics in treatment-refractory schizophrenia: systematic review and meta-analysis. Br J Psychiatry 2016, 209:385392, doi: 10.1192/bjp.bp.115.177261

2. Kar N, Barreto S, Chandavarkar, R. Clozapine Monitoring in Clinical Practice: Beyond the Mandatory Requirement. Clin Psychopharmacol Neurosci 2016, 14:323-329, doi: 10.9758/cpn.2016.14.4.323

3. Jonathan G. Leung, Terri S. Wittenberger, Kathryn M. Schak. Clozapine treated patients and COVID-19: Ensuring continued care through collaboration. Schizophr Res 2020, 222:507-508, doi: 10.1016/j.schres.2020. 05.030

4. Siskind D, Honer WG, Clark S, Correll CU, Hasan A, Howes O et al. Consensus statement on the use of clozapine during the COVID-19 pandemic. J Psychiatry Neurosci 2020, 45:200061, doi: 10.1503/jpn. 200061 


\title{
Clozapine treatment: Ensuring ongoing monitoring during the COVID-19 pandemic
}

\author{
ARTICLE HISTORY: Received 26 January 2021/Revised 2 February 2021/Published Online 17 March 2021
}

\section{Dear Editors,}

The current coronavirus pandemic (COVID-19) has led mental health systems to uncertainty regarding safe continuation of clozapine monitoring protocols. Clozapine is without doubt the only antipsychotic available with repeatedly proven efficacy in treatment resistant schizophrenia. ${ }^{1}$ Replacing clozapine with an alternative antipsychotic in patients stabilized with clozapine can potentially lead to higher risk of relapse or exacerbation of severity of illness. ${ }^{1}$

Clozapine, as already known, has a number of side effects, some of which can be serious, thus patients receiving clozapine require ongoing scheduled monitoring. Side effects of clozapine include neutropenia or agranulocytosis, myocarditis, fever, hypersalivation, weight gain and constipation. These side effects can be detected and treated when recognized on time decreasing the possibility of serious consequences making the implementation of an ongoing treatment monitoring protocol for patients on clozapine mandatory. ${ }^{2}$

Since it was advised for all mental health providers in most countries worldwide to limit non-urgent hospital visits and procedures to reduce the risk of contamination a challenge arose for patients' ability to access health care facilities for their routine clozapine monitoring. Nevertheless, the majority of Mental Health Care Authorities decided to ensure access for all patients on clozapine to their routine monitoring protocol. ${ }^{3,4}$

To date, no data exist on any potential relationship between antipsychotic use and the risk of contamination with SARS-CoV-2 or the development of severe symptoms of the infection. The literature suggests that patients receiving antipsychotics, especially clozapine, have an increased risk of developing pneumonia, leading to the assumption that patients receiving clozapine are at higher risk to develop COVID-19.'

Balancing the importance of monitoring continuation against the increased risk for COVID-19, an International Consensus Statement was recently published addressing a monitoring protocol with reduced visits. The Consensus suggested reduced hematologic monitoring frequency of every 3 months with a prescription of 90 days clozapine supply (if safe). The above applies to patients receiving clozapine for at least one year without neutropenia. The risk of neutropenia after 12 months of clozapine treatment falls significantly. ${ }^{4}$

Based on the above it is suggested to all clozapine clinics to implement a guidance monitoring protocol for all patients on clozapine to ensure safety during the pandemic. Besides hematological monitoring that requires physical contact with healthcare workers it is significant to implement a telemedicine appointment in frequent intervals to monitor symptoms of infection, symptoms of cardiovascular diseases and constipation. Patient should also be advised to regularly monitor one's blood pressure and pulses and ideally be educated on how by a member of the staff. If a patient is detected with any symptoms related to the above an emergency appointment for evaluation should be planned.

Overall, since both the consequences and the duration of the pandemic are unknown, mental health services must work jointly to implement a clozapine monitoring plan to ensure safe continuation in such a vulnerable population

\section{Maria Nystazaki}

Second Department of Psychiatry, National and Kapodistrian University of Athens, "Attikon" University Hospital, Athens, Greece e-mail:manystazaki@nurs.uoa.gr

\section{Giorgos Alevizopoulos}

Department of Psychiatry, Aghioi Anargyroi Hospital, National and Kapodistrian University of Athens, Athens, Greece e-mail:galev@nurs.uoa.gr

\section{References}

1. Siskind D, McCartney L, Goldschlager R, Kisely S. Clozapine v. first- and second-generation antipsychotics in treatment-refractory schizophrenia: systematic review and meta-analysis. Br J Psychiatry 2016, 209:385392, doi: 10.1192/bjp.bp.115.177261

2. Kar N, Barreto S, Chandavarkar, R. Clozapine Monitoring in Clinical Practice: Beyond the Mandatory Requirement. Clin Psychopharmacol Neurosci 2016, 14:323-329, doi: 10.9758/cpn.2016.14.4.323

3. Jonathan G. Leung, Terri S. Wittenberger, Kathryn M. Schak. Clozapine treated patients and COVID-19: Ensuring continued care through collaboration. Schizophr Res 2020, 222:507-508, doi: 10.1016/j.schres.2020. 05.030

4. Siskind D, Honer WG, Clark S, Correll CU, Hasan A, Howes O et al. Consensus statement on the use of clozapine during the COVID-19 pandemic. J Psychiatry Neurosci 2020, 45:200061, doi: 10.1503/jpn. 200061 